\title{
Stability of generic cylindrical thin shell wormholes
}

\author{
S. Habib Mazharimousavi, ${ }^{*}$ M. Halilsoy, ${ }^{\dagger}$ and Z. Amirabi ${ }^{\ddagger}$ \\ Department of Physics, Eastern Mediterranean University, Gazimağusa, North Cyprus, Mersin 10, Turkey
}

(Received 20 February 2014; published 1 April 2014)

\begin{abstract}
We revisit the stability analysis of cylindrical thin-shell wormholes which have been studied in literature so far. Our approach is more systematic and in parallel to the method which is used in spherically symmetric thin-shell wormholes. The stability condition is summarized as the positivity of the second derivative of an effective potential at the equilibrium radius, i.e., $V^{\prime \prime}\left(a_{0}\right)>0$. This may serve as the master equation in all stability problems for the cylindrical thin-shell wormholes.
\end{abstract}

DOI: 10.1103/PhysRevD.89.084003

PACS numbers: 04.20.Jb, 04.20.Gz, 04.40.Nr

\section{INTRODUCTION}

Upon the breaking of spherical symmetry in an axial direction, we arrive at cylindrical symmetry. A large number of systems fail to satisfy spherical symmetry and are considered within the context of cylindrical (or axial) symmetry. Spacetimes that depend on radial $r$ and time $t$ are known to describe cylindrical waves. Replacing $t$ with the spacelike coordinate $z$ gives rise to static, axially symmetric spacetimes. Our interest in this study is to suppress the $t$ and $z$ dependences and consider spacetimes depending only on the radial $r$ coordinate. This amounts to admitting three Killing vectors $\left(\xi_{t}^{\mu}, \xi_{z}^{\mu}\right.$, and $\left.\xi_{\varphi}^{\mu}\right)$ in the Weyl coordinates $\{t, r, z, \varphi\}$. Historically, the first such example was given by Levi-Civita [1]. Topological defect spacetimes believed to form during the early Universe, such as cosmic strings [2], also fit into this class. The latter's currentlike source is located along an axis which creates a deficit angle in the surrounding space so that it gives rise to gravitational lensing. Still another example for cylindrically symmetric metrics, which is powered by a beamlike magnetic field, is Melvin's magnetic universe [3]. The addition of extra fields such as the Brans-Dicke scalar or various electromagnetic fields to the cylindrical metrics has been extensively searched in the literature [4]. Recently, we have given an example of a Weyl solution in which the magnetic Melvin and Bertotti-Robinson metrics are combined in a simple Einstein-Maxwell metric [5]. There is already a large literature related to the spherically symmetric thin-shell wormholes (TSW) [6], but for the cylindrically symmetric cases the published literature is relatively less [7]. From this token, we wish to consider a general class of cylindrically symmetric spacetimes in which the metric functions depend only on the radial function $r$ to construct TSWs. As usual, our method is the cutting and pasting of two cylindrically symmetric spacetimes, which unlike the spherical symmetric cases, are more restrictive toward asymptotic flatness. Being

\footnotetext{
"habib.mazhari@emu.edu.tr mustafa.halilsoy@emu.edu.tr *zahra.amirabi@emu.edu.tr
}

$z$ independent, the metric is same for both $z=0$ and $|z|=\infty$. Yet the areal/radial flare-out conditions must be satisfied [8-11], in spite of the fact that the spacetime may not be asymptotically flat. The radial $\left(P_{r}\right)$ and axial $\left(P_{z}\right)$ pressures are assumed to be functions of the energy (mass) density $\sigma$. The junction conditions at the intersection determine the throat equation as a function of the proper time. From the extrinsic curvature components we extract an energy equation for a one-dimensional particle of the form $\dot{a}^{2}+V(a)=0$, where $a(\tau)$ is the radius of the throat and the dot means a proper time derivative. The form of the potential $V(a)$ can be rather complicated, but since we are interested in the stability, we need to investigate only the second derivative of the potential around the equilibrium radius of the throat. The parametric plotting of the second derivative of the potential $V^{\prime \prime}\left(a_{0}\right)>0$, where $a_{0}$ is the equilibrium radius, reveals the stability region for the TSW under consideration. Our perturbation addresses only the radial and linear cases for which we may adopt equations of state (EOSs) for the surface energy-momentum at the throat. Adding an extra source amounts to the fact that the covariant divergence of the surface energy-momentum is nonzero. The structural equations for perturbations expectedly are more complicated than in the spherical symmetric case, which is natural from the less-symmetry arguments. Concerning the exotic/normal matter, however, our formalism does not add anything new; i.e., our matter to thread the TSW is still exotic. In a recent study, we have proposed that in order to get anything total but exotic matter as source, albeit locally is exotic, the geometry of the throat must be of prolate/oblate type [12].

Organization of the paper is as follows: In Sec. II, we consider a general line element with cylindrical symmetry and derive the stability condition for the TSW. In Sec. III, we make applications of the result found in Sec. II. We complete the paper with a Conclusion in Sec. IV.

\section{GENERAL ANALYSIS FOR A CYLINDRICALLY SYMMETRIC TSW}

Let us consider two static, cylindrically symmetric spacetimes $\mathcal{M}_{ \pm}[8,13]$ in Weyl coordinates: 


$$
\begin{aligned}
d s^{2}= & -e^{2 \gamma_{ \pm}\left(r_{ \pm}\right)} d t_{ \pm}^{2}+e^{2 \alpha_{ \pm}\left(r_{ \pm}\right)} d r_{ \pm}^{2}+e^{2 \xi_{ \pm}\left(r_{ \pm}\right)} d z_{ \pm}^{2} \\
& +e^{2 \beta_{ \pm}\left(r_{ \pm}\right)} d \varphi_{ \pm}^{2} .
\end{aligned}
$$

By gluing these two manifolds at their boundaries $\Sigma_{ \pm}$, one can, in principle, make a single complete manifold. Each separate spacetime $\mathcal{M}_{ \pm}$must satisfy the Einstein equations with a general form of the energy-momentum tensor $T_{\mu \pm}^{\nu}=\left[-\rho_{ \pm}, p_{r \pm}, p_{z \pm}, p_{\varphi \pm}\right]$

$$
G_{\mu \pm}^{\nu}=T_{\mu \pm}^{\nu},
$$

with the unit convention $(8 \pi G=c=1)$. Einstein's equations in each spacetime admit (for simplicity, we suppress sub- \pm for each spacetime, but they are implicitly there)

$$
\begin{gathered}
-\rho=e^{-2 \alpha}\left[\beta^{\prime \prime}+\xi^{\prime \prime}+\beta^{2}+\left(\xi^{\prime}-\alpha^{\prime}\right)\left(\beta^{\prime}+\xi^{\prime}\right)\right], \\
p_{r}=e^{-2 \alpha}\left[\left(\beta^{\prime}+\xi^{\prime}\right) \gamma^{\prime}+\xi^{\prime} \beta^{\prime}\right], \\
p_{z}=-2 \alpha\left[\gamma^{\prime \prime}+\beta^{\prime \prime}+\gamma^{\prime 2}+\left(\gamma^{\prime}+\beta^{\prime}\right)\left(\beta^{\prime}-\alpha^{\prime}\right)\right],
\end{gathered}
$$

and

$$
p_{\varphi}={ }^{-2 \alpha}\left[\gamma^{\prime \prime}+\xi^{\prime \prime}+\gamma^{\prime 2}+\left(\gamma^{\prime}+\xi^{\prime}\right)\left(\xi^{\prime}-\alpha^{\prime}\right)\right],
$$

in which a prime stands for the derivative with respect to $r_{ \pm}$ depending on the manifold under consideration.

After gluing the two spacetimes at their boundaries whose equation in our study is given by $\mathcal{H}=$ $r-a(\tau)=0$, the intrinsic line element on the common boundary $\Sigma=\Sigma_{ \pm}$can be written as

$$
d s_{\Sigma}^{2}=-d \tau^{2}+e^{2 \xi(a)} d z^{2}+e^{2 \beta(a)} d \varphi^{2},
$$

in which $a=a(\tau)$ is a function of proper time $\tau$. The normal four-vector on the timelike hypersurface $\Sigma$ is defined as

$$
n_{\gamma}^{( \pm)}=\left( \pm\left|g^{\alpha \beta} \frac{\partial \mathcal{H}}{\partial x^{\alpha}} \frac{\partial \mathcal{H}}{\partial x^{\beta}}\right|^{-1 / 2} \frac{\partial \mathcal{H}}{\partial x^{\gamma}}\right)_{\Sigma}
$$

which in closed form becomes

$$
n_{\gamma}^{( \pm)}= \pm\left(-e^{\alpha_{ \pm}+\gamma_{ \pm}} \dot{a}, e^{2 \alpha_{ \pm}} \sqrt{\Delta_{ \pm}}, 0,0\right)_{\Sigma},
$$

where $\Delta_{ \pm}=e^{-2 \alpha_{ \pm}}+\dot{a}^{2}$ and a dot stands for the derivative with respect to the proper time. Next, we find the extrinsic curvature on the hypersurface $\Sigma$, defined as

$$
K_{i j}^{( \pm)}=-n_{\gamma}^{( \pm)}\left(\frac{\partial^{2} x_{ \pm}^{\gamma}}{\partial X_{ \pm}^{i} \partial X_{ \pm}^{j}}+\Gamma_{ \pm \alpha \beta}^{\gamma} \frac{\partial x_{ \pm}^{\alpha}}{\partial X_{ \pm}^{i}} \frac{\partial x_{ \pm}^{\beta}}{\partial X_{ \pm}^{j}}\right)_{\Sigma},
$$

in which $X_{ \pm}^{i} \in\left\{\tau, z_{ \pm}, \varphi_{ \pm}\right\}$, while $x_{ \pm}^{\gamma}=\left\{t_{ \pm}, r_{ \pm}, z_{ \pm}, \varphi_{ \pm}\right\}$. Explicit calculations yield

$$
\begin{gathered}
K_{\tau}^{\tau( \pm)}= \pm\left(\frac{1}{\sqrt{\Delta_{ \pm}}}\left(\ddot{a}+\left(\alpha_{ \pm}^{\prime}+\gamma_{ \pm}^{\prime}\right) \dot{a}^{2}+e^{-2 \alpha_{ \pm}} \gamma_{ \pm}^{\prime}\right)\right)_{\Sigma}, \\
K_{z}^{z( \pm)}= \pm\left(\xi_{ \pm}^{\prime} \sqrt{\Delta_{ \pm}}\right)_{\Sigma},
\end{gathered}
$$

and

$$
K_{\varphi}^{\varphi( \pm)}= \pm\left(\beta_{ \pm}^{\prime} \sqrt{\Delta_{ \pm}}\right)_{\Sigma} .
$$

By considering a standard energy-momentum on the shell, i.e., $S_{i}^{j}=\operatorname{diag}\left(-\sigma, P_{z}, P_{\varphi}\right)$, the Israel junction conditions [14] imply

$$
\left\langle K_{i}^{j}\right\rangle-\langle K\rangle \delta_{i}^{j}=-S_{i}^{j},
$$

in which $\left\langle K_{i}^{j}\right\rangle=\left\langle K_{i}^{j}\right\rangle_{+}-\left\langle K_{i}^{j}\right\rangle_{-}$and $\langle K\rangle=\left\langle K_{i}^{i}\right\rangle$. The latter amounts to

$$
\begin{aligned}
\sigma=-\left[\left(\xi_{+}^{\prime}+\beta_{+}^{\prime}\right) \sqrt{\Delta_{+}}+\left(\xi_{-}^{\prime}+\beta_{-}^{\prime}\right) \sqrt{\Delta_{-}}\right], \\
P_{z}=\frac{\left(\ddot{a}+\left(\alpha_{+}^{\prime}+\gamma_{+}^{\prime}\right) \dot{a}^{2}+e^{-2 \alpha_{+}} \gamma_{+}^{\prime}\right)}{\sqrt{\Delta_{+}}} \\
+\frac{\left(\ddot{a}+\left(\alpha_{-}^{\prime}+\gamma_{-}^{\prime}\right) \dot{a}^{2}+e^{-2 \alpha_{-} \gamma_{-}^{\prime}}\right)}{\sqrt{\Delta_{-}}} \\
+\beta_{+}^{\prime} \sqrt{\Delta_{+}}+\beta_{-}^{\prime} \sqrt{\Delta_{-}}
\end{aligned}
$$

and

$$
\begin{aligned}
P_{\varphi}= & \frac{\left(\ddot{a}+\left(\alpha_{+}^{\prime}+\gamma_{+}^{\prime}\right) \dot{a}^{2}+e^{-2 \alpha_{+}} \gamma_{+}^{\prime}\right)}{\sqrt{\Delta_{+}}} \\
& +\frac{\left(\ddot{a}+\left(\alpha_{-}^{\prime}+\gamma_{-}^{\prime}\right) \dot{a}^{2}+e^{-2 \alpha_{-}} \gamma_{-}^{\prime}\right)}{\sqrt{\Delta_{-}}} \\
& +\xi_{+}^{\prime} \sqrt{\Delta_{+}}+\xi_{-}^{\prime} \sqrt{\Delta_{-}} .
\end{aligned}
$$

To complete this section, we consider a thin shell on the junction which is constructed by the same bulk spacetime, so that on the boundaries $\gamma, \alpha, \beta$, and $\xi$ are continuous, and consequently

$$
\begin{gathered}
\sigma=-2\left(\xi^{\prime}+\beta^{\prime}\right) \sqrt{\Delta}, \\
P_{z}=\frac{2\left(\ddot{a}+\left(\alpha^{\prime}+\gamma^{\prime}\right) \dot{a}^{2}+e^{-2 \alpha} \gamma^{\prime}\right)}{\sqrt{\Delta}}+2 \beta^{\prime} \sqrt{\Delta},
\end{gathered}
$$

and

$$
P_{\varphi}=\frac{2\left(\ddot{a}+\left(\alpha^{\prime}+\gamma^{\prime}\right) \dot{a}^{2}+e^{-2 \alpha} \gamma^{\prime}\right)}{\sqrt{\Delta}}+2 \xi^{\prime} \sqrt{\Delta}
$$

From now on, our study will be concentrated on the case of a TSW made from a single bulk metric. 


\section{A. Energy conservation identity}

The energy conservation identity can be found by calculating $S_{; j}^{i j}$. Our explicit calculations show that

$$
S_{; j}^{i j i=\tau}=\frac{d \sigma}{d \tau}+\dot{a}\left(\xi^{\prime}+\beta^{\prime}\right) \sigma+\dot{a}\left(\xi^{\prime} P_{z}+\beta^{\prime} P_{\varphi}\right) .
$$

Furthermore, when we consider the exact forms of $\sigma, P_{z}$, and $P_{\varphi}$, we find from the latter

$$
\frac{d(\mathcal{A} \sigma)}{d \tau}+e^{\beta} P_{z} \frac{d\left(e^{\xi}\right)}{d \tau}+e^{\xi} P_{\varphi} \frac{d\left(e^{\beta}\right)}{d \tau}=\dot{a} \mathcal{A} \Xi,
$$

in which

$$
\Xi=\sigma\left[\frac{\beta^{2}+\xi^{\prime 2}+\beta^{\prime \prime}+\xi^{\prime \prime}}{\beta^{\prime}+\xi^{\prime}}-\left(\alpha^{\prime}+\gamma^{\prime}\right)\right]
$$

and the surface area of the shell $\mathcal{A}=e^{\beta+\xi}$. In Eq. (22), $\frac{d(\mathcal{A} \sigma)}{d \tau}$ is the time change of the total internal energy of the shell, and $e^{\beta} P_{z} \frac{d\left(e^{\xi}\right)}{d \tau}, e^{\xi} P_{\varphi} \frac{d\left(e^{\beta}\right)}{d \tau}$, and $\dot{a} \mathcal{A} \Xi$ are the work done in the $z, \varphi$ directions and the external forces, respectively. This is comparable with the similar result in spherically symmetric TSWs given in Ref. [13].

\section{B. Stability of the thin-shell wormhole}

In this section, we apply a linear perturbation and investigate whether the wormhole is stable against the perturbation analysis or not. Our main assumption is that the matter which supports the TSW obeys the energy conservation identity. This in turn implies that from Eq. (22),

$$
(\mathcal{A} \sigma)^{\prime}+e^{\beta} P_{z}\left(e^{\xi}\right)^{\prime}+e^{\xi} P_{\varphi}\left(e^{\beta}\right)^{\prime}=\mathcal{A} \Xi,
$$

in which a prime stands for the derivative with respect to $a$. Following our linear perturbation, the wormhole is dynamic, and from Eq. (18), one finds the equation of the throat as a one-dimensional motion $\dot{a}^{2}+V(a)=0$ with potential

$$
V(a)=e^{-2 \alpha}-\frac{1}{4}\left(\frac{\sigma}{\xi^{\prime}+\beta^{\prime}}\right)^{2} \text {. }
$$

If $a=a_{0}$ is considered as an equilibrium point with $\dot{a}_{0}=0=\ddot{a}_{0}$, then $V(a)$ can be expanded about the equilibrium point at which $V\left(a_{0}\right)=0=V^{\prime}\left(a_{0}\right)$. Also, the components of the energy-momentum tensor on the shell when the equilibrium state is considered are given by

$$
\begin{gathered}
\sigma_{0}=-2\left(\xi_{0}^{\prime}+\beta_{0}^{\prime}\right) e^{-\alpha_{0}}, \\
P_{z 0}=2\left(\gamma_{0}^{\prime}+\beta_{0}^{\prime}\right) e^{-\alpha_{0}},
\end{gathered}
$$

and

$$
P_{\varphi 0}=2\left(\gamma_{0}^{\prime}+\xi_{0}^{\prime}\right) e^{-\alpha_{0}}
$$

We note that a sub- 0 notation implies that the corresponding quantity is calculated at the equilibrium point. Next, we find $V^{\prime \prime}\left(a_{0}\right)=V_{0}^{\prime \prime}$ to examine the motion of the throat. If $V_{0}^{\prime \prime}>0$, then the motion is oscillatory and the equilibrium at $a_{0}$ is stable; otherwise, it is unstable. To find $V^{\prime \prime}$, we need $\sigma^{\prime}$ and $\sigma^{\prime \prime}$, which are given by the energy conservation identity, i.e.,

$$
\sigma^{\prime}=\Xi-P_{z} \xi^{\prime}-P_{\varphi} \beta^{\prime}-\left(\beta^{\prime}+\xi^{\prime}\right) \sigma
$$

and

$$
\begin{aligned}
\sigma^{\prime \prime}= & \Xi^{\prime}-P_{z}^{\prime} \xi^{\prime}-P_{z} \xi^{\prime \prime}-P_{\varphi}^{\prime} \beta^{\prime}-P_{\varphi} \beta^{\prime \prime}-\left(\beta^{\prime \prime}+\xi^{\prime \prime}\right) \sigma \\
& -\left(\beta^{\prime}+\xi^{\prime}\right)\left(\Xi-P_{z} \xi^{\prime}-P_{\varphi} \beta^{\prime}-\left(\beta^{\prime}+\xi^{\prime}\right) \sigma\right) .
\end{aligned}
$$

Our extensive calculation eventually yields

$$
\begin{aligned}
V_{0}^{\prime \prime}= & -\frac{2 e^{-2 \alpha_{0}}}{\beta_{0}^{\prime}+\xi_{0}^{\prime}}\left[\beta_{0}^{\prime 2} \phi_{0}^{\prime} \alpha_{0}^{\prime}+\left(\alpha_{0}^{\prime}\left[\phi_{0}^{\prime}+\psi_{0}^{\prime}+2\right] \xi_{0}^{\prime}+\alpha_{0}^{\prime} \gamma_{0}^{\prime}-\left[\beta_{0}^{\prime \prime}+\xi_{0}^{\prime \prime}\right] \phi_{0}^{\prime}-\xi_{0}^{\prime \prime}-\gamma_{0}^{\prime \prime}\right) \beta_{0}^{\prime}\right. \\
& \left.+\left(\xi_{0}^{\prime} \psi_{0}^{\prime} \alpha_{0}^{\prime}+\alpha_{0}^{\prime} \gamma_{0}^{\prime}-\left(\beta_{0}^{\prime \prime}+\xi_{0}^{\prime \prime}\right) \psi_{0}^{\prime}-\gamma_{0}^{\prime \prime}-\beta_{0}^{\prime \prime}\right) \xi_{0}^{\prime}\right] .
\end{aligned}
$$

Here in this expression, the EOS is considered to be $P_{z}=\psi(\sigma), P_{\varphi}=\phi(\sigma)$. We also note that a prime on a function denotes the derivative with respect to its argument -for instance, $\psi_{0}^{\prime}=\left.\frac{\partial \psi}{\partial \sigma}\right|_{\sigma=\sigma_{0}}$, while $\beta_{0}^{\prime}=\left.\frac{\partial \beta}{\partial a}\right|_{a=a_{0}}$. Having the form of the metric functions and the EOS is enough to check whether the TSW is stable or not.

Before we proceed to examine the stability of the TSW, we would like to introduce the conditions which should be satisfied for having a wormhole in cylindrical symmetry. These conditions were studied in Ref. [8], where the first condition is called the areal flare-out condition, stating that $e^{\xi+\beta}$ must be an increasing function at the throat [8-11].
The second condition implies that $e^{\beta}$ must be an increasing function at the throat and is called the radial flare-out condition [8-11]. According to Ref. [8], the appropriate condition would be the radial flare-out condition.

\section{The Levi-Civita metric}

Before we find some applications for the general equation [Eq. (31)] among the known cylindrical TSWs in the literature, we give the simplest cylindrical TSW which can be made in the vacuum Levi-Civita (LC) metric [1]. The LC metric with two essential parameters $b$ and $\delta$ can be written as 


$$
d s^{2}=-b r^{4 \delta} d t^{2}+r^{4 \delta(2 \delta-1)}\left(d r^{2}+d z^{2}\right)+\frac{r^{2(1-2 \delta)}}{b} d \varphi^{2},
$$

in which $b$ is related to the topology of the spacetime giving rise to a deficit angle $\theta=2 \pi\left(1-\frac{1}{\sqrt{b}}\right)$ [15]. For a physical interpretation of $\delta$, we refer to the third and fourth papers in Ref. [1]. Comparing the LC line element with our general line element [Eq. (1)], we find that

$$
e^{2 \gamma}=b r^{4 \delta}, \quad e^{2 \alpha}=e^{2 \xi}=r^{4 \delta(2 \delta-1)}, \quad e^{2 \beta}=\frac{r^{2(1-2 \delta)}}{b} .
$$

Once more, we note that in the cut-paste method, we consider two copies of the bulk spacetime (here LC) in which from each we cut the region $r<a(\tau)$, and then we join them at $r=a(\tau)$ to have a complete manifold. Therefore, the outer region of the wormhole is still LC spacetime with the mentioned essential parameters. Furthermore, the radial flare-out condition is satisfied only for $\delta \leq \frac{1}{2}$, while the areal flare-out condition is satisfied for all $\delta$. Considering the TSW at $r=a(\tau)$ and using the general condition of stability-i.e., $V_{0}^{\prime \prime}>0$ together with a linear $\operatorname{EOS} \psi^{\prime}(\sigma)=\phi^{\prime}(\sigma)=\eta_{0}$ in which $\eta_{0}$ is a constant-we find

$$
\left(-2 \eta_{0} \delta^{2}+\left(2 \eta_{0}+1\right) \delta-\frac{\eta_{0}}{2}\right)\left(\delta^{2}-\frac{1}{2} \delta+\frac{1}{4}\right) \geq 0 .
$$

In Fig. 1, we plot the stability region in terms of the parameters $\delta$ and $\eta_{0}$, and as can be observed from the figure, the stability is sensitive with respect to $\delta$. In particular, for $\delta<\frac{1}{4}$ the stability is not strong enough, while for $\frac{1}{4}<\delta<1$ it is quite strong. Note that the topological parameter $b$ does not play a role in the stability of the LC wormhole.

\section{APPLICATIONS}

Eiroa and Simeone in Ref. [9] have considered a general static cylindrical metric in $3+1$ dimensions given by

$$
d s^{2}=B(r)\left(-d t^{2}+d r^{2}\right)+C(r) d \varphi^{2}+D(r) d z^{2},
$$

in which $B(r), C(r)$, and $D(r)$ are only functions of $r$. Using the results found above together with $e^{2 \alpha}=e^{2 \gamma}=B$, $e^{2 \beta}=C$, and $e^{2 \xi}=D$, one finds

$$
\begin{gathered}
\sigma=-\left(\frac{D^{\prime}}{D}+\frac{C^{\prime}}{C}\right) \sqrt{\Delta}, \\
P_{z}=\frac{1}{\sqrt{\Delta}}\left[2 \ddot{a}+\frac{2 B^{\prime}}{B} \dot{a}^{2}+\frac{B^{\prime}}{B^{2}}+\frac{C^{\prime}}{C} \Delta\right],
\end{gathered}
$$

and

$$
P_{\varphi}=\frac{1}{\sqrt{\Delta}}\left[2 \ddot{a}+\frac{2 B^{\prime}}{B} \dot{a}^{2}+\frac{B^{\prime}}{B^{2}}+\frac{D^{\prime}}{D} \Delta\right] .
$$

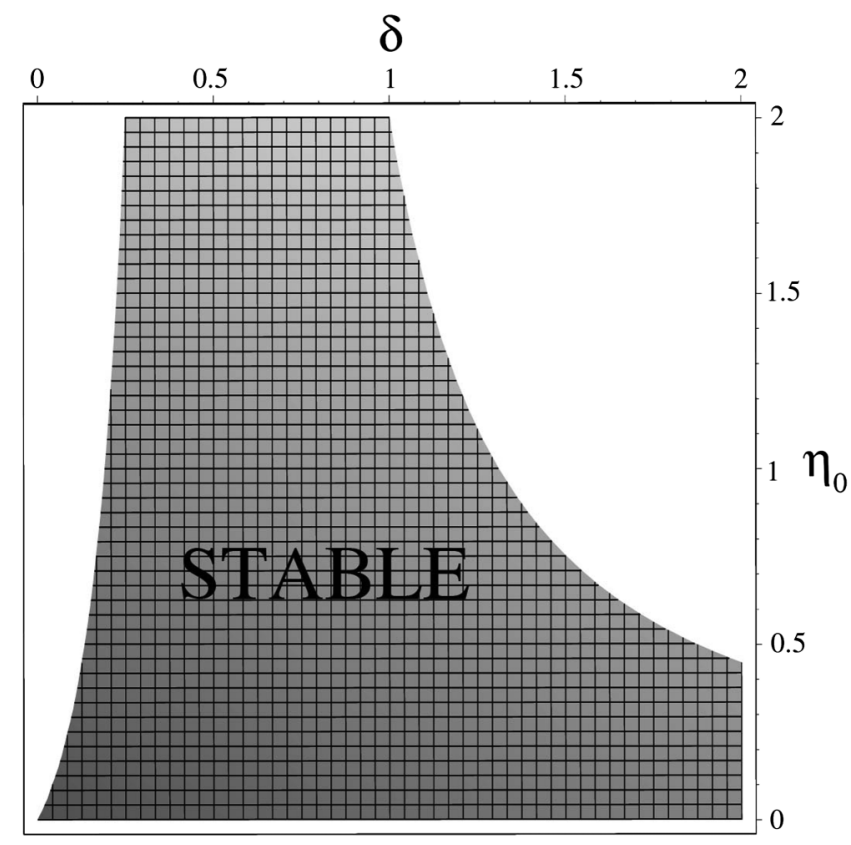

FIG. 1. Stability of a TSW in LC spacetime, supported by a linear gas, in terms of $\delta$ and $\eta_{0}$. We see that the stability depends on one of the essential parameters; i.e., $\delta$ in the LC metric. Also, the radial flare-out condition is satisfied only for $\delta \leq \frac{1}{2}$.

At the equilibrium surface, i.e., $a=a_{0}$, we have

$$
\begin{gathered}
\sigma_{0}=-\left(\frac{D_{0}^{\prime}}{D_{0}}+\frac{C_{0}^{\prime}}{C_{0}}\right) \frac{1}{\sqrt{B_{0}}}, \\
P_{z 0}=\sqrt{B_{0}}\left[2 \ddot{a}+\frac{2 B_{0}^{\prime}}{B_{0}} \dot{a}^{2}+\frac{B_{0}^{\prime}}{B_{0}^{2}}+\frac{C_{0}^{\prime}}{B_{0} C_{0}}\right],
\end{gathered}
$$

and

$$
P_{\varphi}=\sqrt{B_{0}}\left[2 \ddot{a}+\frac{2 B_{0}^{\prime}}{B_{0}} \dot{a}^{2}+\frac{B_{0}^{\prime}}{B_{0}^{2}}+\frac{D_{0}^{\prime}}{B_{0} D_{0}}\right] .
$$

The energy conservation identity becomes

$$
\begin{aligned}
\left(S_{; j}^{i j i=\tau}\right) \frac{d \sigma}{d \tau} & +\left(\frac{D^{\prime}}{2 D}\left(P_{z}+\sigma\right)+\frac{C^{\prime}}{2 C}\left(P_{\varphi}+\sigma\right)\right) \frac{d a}{d \tau} \\
& =-\frac{d a}{d \tau} \sigma\left[\frac{B^{\prime}}{B}-\frac{\zeta}{2}-\frac{\zeta^{\prime}}{\zeta}+\frac{D^{\prime} C^{\prime}}{\zeta D C}\right],
\end{aligned}
$$

in which

$$
\zeta=\frac{D^{\prime}}{D}+\frac{C^{\prime}}{C}
$$

The potential of the motion of the throat $V(a)$ reduces to

$$
V(a)=\frac{1}{B}-\left(\frac{\sigma}{\zeta}\right)^{2}
$$

whose second derivative at point $a=a_{0}$ becomes 


$$
\begin{aligned}
V_{0}^{\prime \prime}= & \frac{C_{0}^{\prime}\left\{\left[\left(2 B_{0} D_{0}^{\prime \prime}-B_{0}^{\prime} D_{0}^{\prime}\right) D_{0}-2 B_{0} D_{0}^{\prime 2}\right] C_{0}^{2}+D_{0}^{2}\left(2 B_{0} C_{0}^{\prime \prime}-B_{0}^{\prime} C_{0}^{\prime}\right) C_{0}-2 D_{0}^{2} C_{0}^{\prime 2} B_{0}\right\}}{2 B_{0}^{2}\left(D_{0}^{\prime} C_{0}+C_{0}^{\prime} D_{0}\right) C_{0}^{2}} \phi_{0}^{\prime} \\
& +\frac{D_{0}^{\prime}\left\{\left[\left(2 D_{0} D_{0}^{\prime \prime}-2 D_{0}^{\prime 2}\right) C_{0}^{2}+2 D_{0}^{2} C_{0} C_{0}^{\prime \prime}-2 D_{0}^{2} C_{0}^{\prime 2}\right] B_{0}-C_{0} D_{0} B_{0}^{\prime}\left(D_{0}^{\prime} C_{0}+C_{0}^{\prime} D_{0}\right)\right\}}{2 C_{0} B_{0}^{2}\left(D_{0}^{\prime} C_{0}+C_{0}^{\prime} D_{0}\right) D_{0}^{2}} \psi_{0}^{\prime} \\
& +\frac{2 D_{0}\left(B_{0} B_{0}^{\prime \prime}-\frac{3}{2} B_{0}^{\prime 2}\right) D_{0}^{\prime} C_{0}^{2}-2 B_{0}^{2} D_{0} C_{0}^{\prime 2} D_{0}^{\prime}}{2 D_{0} C_{0} B_{0}^{3}\left(D_{0}^{\prime} C_{0}+C_{0}^{\prime} D_{0}\right)} \\
& +\frac{C_{0}\left[\left(2 B_{0} B_{0}^{\prime \prime} C_{0}^{\prime}-3 C_{0}^{\prime} B_{0}^{\prime 2}\right) D_{0}^{2}+\left(\left[2 C_{0}^{\prime \prime} D_{0}^{\prime}+2 D_{0}^{\prime \prime} C_{0}^{\prime}\right] B_{0}^{2}-2 C_{0}^{\prime} B_{0} B_{0}^{\prime} D_{0}^{\prime}\right) D_{0}-2 C_{0}^{\prime} B_{0}^{2} D_{0}^{\prime 2}\right]}{2 D_{0} C_{0} B_{0}^{3}\left(D_{0}^{\prime} C_{0}+C_{0}^{\prime} D_{0}\right)},
\end{aligned}
$$

in which all functions are calculated at $a=a_{0}$, while $\psi_{0}^{\prime}=$ $\left.\frac{d \psi}{d \sigma}\right|_{\sigma_{0}}$ and $\phi_{0}^{\prime}=\left.\frac{d \phi}{d \sigma}\right|_{\sigma_{0}}$.

\section{A. Stability of the cylindrical TSW with a positive cosmological constant}

In Ref. [11], Richarte introduced a cylindrical wormhole based on the spacetime in the presence of a cosmic string in vacuum and outside the core of the string, which means $r>r_{\text {core }}$, where the bulk metric functions are given by (for a detailed work see Ref. [11])

$$
\begin{gathered}
B(a)=\cos ^{\frac{4}{3}} \tilde{a}, \\
C(a)=\frac{4 \delta^{2}}{3 \Lambda} \frac{\sin ^{2} \tilde{a}}{\cos ^{\frac{2}{3}} \tilde{a}},
\end{gathered}
$$

and

$$
D(a)=1 .
$$

Here $\tilde{a}=\frac{\sqrt{3 \Lambda}}{2} a, \delta$ is a parameter related to the deficit angle explicitly given in Ref. [16], and $\Lambda$ is the cosmological constant.

An explicit calculation of $V_{0}^{\prime \prime}$ yields

$$
\begin{aligned}
V_{0}^{\prime \prime}= & -\frac{2 \Lambda}{3 \cos ^{\frac{10}{3}} \tilde{a}_{0} \sin ^{2} \tilde{a}_{0}} \\
& {\left[\left(\beta_{2}+1\right) \sin ^{4} \tilde{a}_{0}+\frac{3}{2}\left(1-3 \beta_{2}\right) \sin ^{2} \tilde{a}_{0}+\frac{9}{4} \beta_{2}\right] . }
\end{aligned}
$$

The EOS is a linear gas (LG) in which $\psi^{\prime}(\sigma)=\beta_{1}$ and $\phi^{\prime}(\sigma)=\beta_{2}$, where $\beta_{1}$ and $\beta_{2}$ are two constant parameters. In order to have a stable TSW, $V_{0}^{\prime \prime}$ must be positive. With a positive cosmological constant, ultimately, the condition of stability reduces to

$$
\left(\beta_{2}+1\right) \sin ^{4} \tilde{a}_{0}+\frac{3}{2}\left(1-3 \beta_{2}\right) \sin ^{2} \tilde{a}_{0}+\frac{9}{4} \beta_{2}<0 .
$$

In Fig. 2, we show the regions of stability in a frame of $\beta_{2}$ versus $\tilde{a}$.

\section{B. Stability of the Brans-Dicke cylindrical TSW}

In Ref. [10], Eiroa and Simone presented a TSW in Einstein-Brans-Dicke (EBD) theory. The corresponding metric functions are given by

$$
\begin{gathered}
B(a)=a^{2 d(d-n)+[\omega(n-1)+2 n](n-1),} \\
C(a)=W_{0}^{2} a^{2(n-d),}
\end{gathered}
$$

and

$$
D(a)=a^{2 d} .
$$

Herein, $d$ and $n$ are integration constants such that the scalar field of the BD theory is given in terms of $n$ as

$$
\phi=\phi_{0} a^{1-n} .
$$

Also, $\omega>-3 / 2$ is a free parameter in BD theory while $W_{0} \in \mathbb{R}$. To study the stability of the TSW in this framework, we again consider a LG for the EOS, which means $\psi^{\prime}=\beta_{1}$ and $\phi^{\prime}=\beta_{2}$. The master equation [Eq. (45)] admits

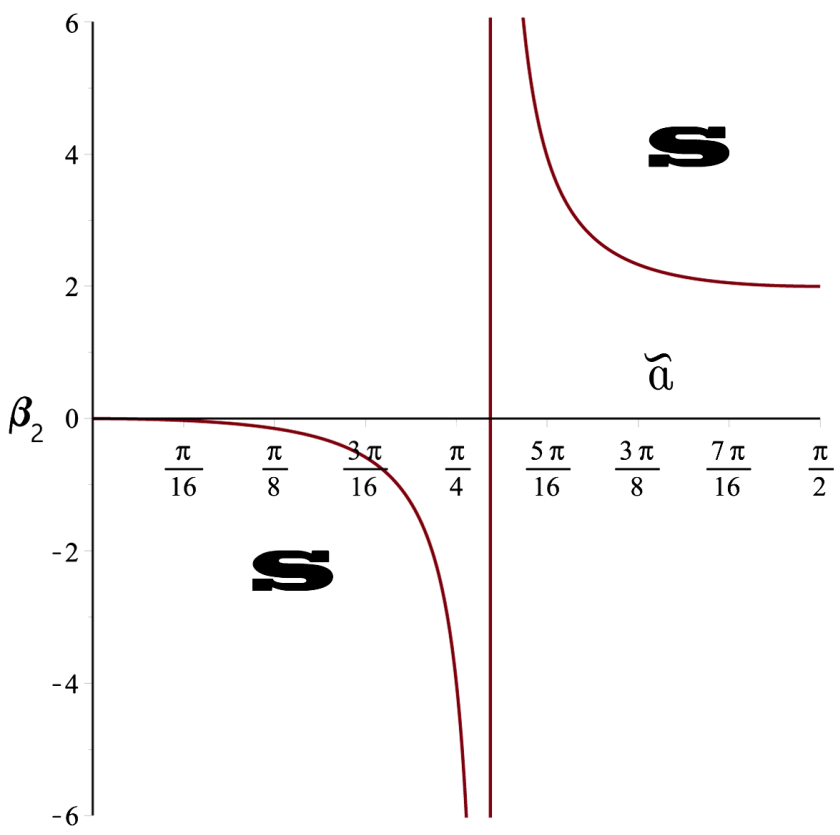

FIG. 2 (color online). Stability of a TSW supported by a LG in terms of $\tilde{a}_{0}$ and $\beta_{2}$. This figure is particularly from Eq. (50). 


$$
V_{0}^{\prime \prime}=\frac{2\left(\frac{\Omega}{2}+1+d(d-n)\right)\left[\left(d-\beta_{2}\right) n^{2}+\left(\left[\beta_{2}-\beta_{1}-2\right] d-\frac{\Omega}{2}-d^{2}\right) n+2 d^{2}\right]}{n a_{0}^{2 d(d-n)+\Omega+2}},
$$

in which $\Omega=[\omega(n-1)+2 n](n-1)$. Imposing $V_{0}^{\prime \prime}>0$ is equivalent to

$$
\left(\frac{\Omega}{2}+1+d(d-n)\right)\left[\left(d-\beta_{2}\right) n^{2}+\left(\left[\beta_{2}-\beta_{1}-2\right] d-\frac{\Omega}{2}-d^{2}\right) n+2 d^{2}\right]>0 .
$$

This final form of the stability involves too many free parameters, which always renders it possible to find some set(s) of parameters to make the TSW stable. In this particular case, one can go further to find a more specific relation.

\section{BD solution with a magnetic field}

In Ref. [10], in addition to the vacuum metric, the authors considered the TSWs in a cylindrically symmetric BD solution with a magnetic field which was introduced in Ref. [17]. Based on Refs. [10,17], the metric functions are given by

$$
\begin{gathered}
B(a)=a^{2 d(d-n)+\Omega}\left(1+c^{2} a^{-2 d+n+1}\right)^{2}, \\
C(a)=\frac{W_{0}^{2} a^{2(n-d)}}{\left(1+c^{2} a^{-2 d+n+1}\right)^{2}},
\end{gathered}
$$

and

$$
D(a)=a^{2 d}\left(1+c^{2} a^{-2 d+n+1}\right)^{2} .
$$

As before, $d$ and $n$ are two integration constants, and $c$ represents the magnetic field strength. In the case of BD with the magnetic field, the areal flare-out condition is trivially satisfied, but to satisfy the radial flare-out condition, one must consider $c^{2}(d-1) a^{-2 d+n+1}+n-d>0$. Clearly, when $c=0$, we get the conditions for the vacuum solution, which becomes $n>d$. Keeping in mind these conditions, we impose $V_{0}^{\prime \prime}>0$. This in turn yields a very complicated expression which we refrain to add here, but instead we remark that for a case with $d=n=1$ and $\beta_{1}=$ $\beta_{2}=\beta$ it becomes

$$
V_{0}^{\prime \prime}=-\frac{2 \beta}{a^{2}\left(1+c^{2}\right)^{2}},
$$

which is clearly positive if $\beta<0$. We note that with our specific setting, only the areal flare-out condition is satisfied, leaving the radial flare-out condition open.

\section{CONCLUSION}

TSWs are considered in cylindrical symmetry where the metric functions rely entirely on the radial Weyl coordinate. Such spacetimes may not be asymptotically flat in general, so we expect deviations from the spherically symmetric counterparts. The source to support the TSW is exotic. Stability analysis in radial direction is worked out in detail, and a master equation is obtained for an effective potential. This is summarized as $V^{\prime \prime}\left(a_{0}\right)>0$, which turns out to be a tedious equation for a generic cylindrically symmetric metric. For specific examples, however, such as LeviCivita, Brans-Dicke with magnetic fields, and similar cases, the stability equation becomes tractable. Parametric plots of the stability regions can be obtained without much effort. Since our case is a generic one, all known cylindrically symmetric TSW solutions to date can be cast into our format. Finally we would like to add that in this work we have only considered the EOS of the fluid which supports the TSW to be a LG. Other possibilities which have been considered so far for the spherical cases, such as Chaplygin gas (CG), generalized Chaplygin gas (GCG), modified generalized Chaplygin gas (MGCG), and logarithmic gas (LG), are open problems to be considered [18].
[1] T. Levi-Civita, Rend. Acc. Lincei 26, 307 (1917); M. F. A. da Silva, L. Herrera, F. M. Paiva, and N. O. Santos, J. Math. Phys. (N.Y.) 36, 3625 (1995); L. Herrera, J. Ruifernandez, and N. O. Santos, Gen. Relativ. Gravit. 33, 515 (2001); L. Herrera, N. O. Santos, A. F. F. Teixeira, and A. Z. Wang, Classical Quantum Gravity 18, 3847 (2001).
[2] A. Vilenkin, Phys. Rep. 121, 263 (1985); Phys. Rev. D 23, 852 (1981); W. A. Hiscock, Phys. Rev. D 31, 3288 (1985).

[3] M. A. Melvin, Phys. Lett. 8, 65 (1964); W. B. Bonnor, Proc. Phys. Soc. London Sect. A 67, 225 (1954); D. Garfinkle and E. N. Glass, Classical Quantum Gravity 28, 215012 (2011). 
[4] C. H. Brans and R. H. Dicke, Phys. Rev. 124, 925 (1961); A. Arazi and C. Simeone, Gen. Relativ. Gravit. 32, 2259 (2000).

[5] S. H. Mazharimousavi and M. Halilsoy, Phys. Rev. D 88, 064021 (2013).

[6] M. Visser, Phys. Rev. D 39, 3182 (1989); Nucl. Phys. B328, 203 (1989); P. R. Brady, J. Louko and E. Poisson, Phys. Rev. D 44, 1891 (1991); E. Poisson and M. Visser, Phys. Rev. D 52, 7318 (1995); M. Ishak and K. Lake, Phys. Rev. D 65, 044011 (2002); C. Simeone, Int. J. Mod. Phys. D 21, 1250015 (2012); F. S. N. Lobo, Phys. Rev. D 71, 124022 (2005); E. F. Eiroa and C. Simeone, Phys. Rev. D 71, 127501 (2005); E. F. Eiroa, Phys. Rev. D 78, 024018 (2008); F. S. N. Lobo and P. Crawford, Classical Quantum Gravity 22, 4869 (2005); S. H. Mazharimousavi, M. Halilsoy, and Z. Amirabi, Phys. Lett. A 375, 3649 (2011); M. Sharif and M. Azam, Eur. Phys. J. C 73, 2407 (2013); 732554 (2013); S. H. Mazharimousavi and M. Halilsoy, Eur. Phys. J. C 73, 2527 (2013).

[7] E. F. Eiroa and C. Simeone, Phys. Rev. D 70, 044008 (2004); M. Sharif and M. Azam, J. Cosmol. Astropart. Phys. 04 (2013) 023; E. Rubín de Celis, O. P. Santillan, and C. Simeone, Phys. Rev. D 86, 124009 (2012); C. Bejarano, E. F. Eiroa, and C. Simeone, Phys. Rev. D 75, 027501 (2007); K. A. Bronnikov, V. G. Krechet, and J. P. S. Lemos,
Phys. Rev. D 87, 084060 (2013); M. G. Richarte, Phys. Rev. D 87, 067503 (2013).

[8] K. A. Bronnikov and J. P. S. Lemos, Phys. Rev. D 79, 104019 (2009).

[9] E. F. Eiroa and C. Simeone, Phys. Rev. D 81, 084022 (2010).

[10] E. F. Eiroa and C. Simeone, Phys. Rev. D 82, 084039 (2010).

[11] M. G. Richarte, Phys. Rev. D 88, 027507 (2013).

[12] S. H. Mazharimousavi and M. Halilsoy, arXiv:1311.6697.

[13] N. M. Garcia, F. S. N. Lobo, and M. Visser, Phys. Rev. D 86, 044026 (2012).

[14] W. Israel, Nuovo Cimento B 44, 1 (1966); V. de la Cruz and W. Israel, Nuovo Cimento A 51, 774 (1967); J. E. Chase, Nuovo Cimento B 67, 136. (1970); S. K. Blau, E. I. Guendelman, and A.H. Guth, Phys. Rev. D 35, 1747 (1987); R. Balbinot and E. Poisson, Phys. Rev. D 41, 395 (1990).

[15] J. S. Dowker, Nuovo Cimento B 52, 129 (1967).

[16] S. Bhattacharya and A. Lahiri, Phys. Rev. D 78, 065028 (2008).

[17] A. Baykal and O. Delice, Gen. Relativ. Gravit. 41, 267 (2009).

[18] Z. Amirabi, M. Halilsoy, and S. H. Mazharimousavi, Phys. Rev. D 88, 124023 (2013). 